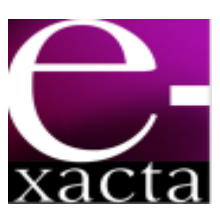

ISSN: 1984-3151

\section{UTILIZAÇÃO DA TERMOGRAFIA INFRAVERMELHA NA DETERMINAÇÃO DE PROPRIEDADES TÉRMICAS DE MATERIAIS PRODUZIDOS A PARTIR DE FIBRA DE BANANEIRA}

\author{
USE OF INFRARED TERMOGRAPHY FOR DETERMINATION OF THERMAL \\ PROPERTIES OF MATERIALS PRODUCED FROM FIBER BANANA PLANT
}

\begin{abstract}
Sinthya Gonçalves Tavares ${ }^{1}$, Maria Elena Walter ${ }^{2}$, Vitório Delogo de Castro ${ }^{2}$, Rogério Alexandre Melo ${ }^{2}$, Christianne Garcia Rodrigues ${ }^{2}$, Mário Carlos de Barros Júnior ${ }^{2}$, Júlio César Rodrigues Cardoso; Sabrina Pinheiro Martins Franco²; Guilherme Fonseca Mendes $^{2}$; Marcela de Oliveira Dias Carneiro²; Fernanda Cunha Silveira ${ }^{2}$
\end{abstract}

Recebido em: 30/10/2011 - Aprovado em: 03/12/2011 - Disponibilizado em: 30/12/2011

RESUMO: Por ser um dos maiores produtores mundiais de banana, o Brasil também é um grande gerador de resíduos provenientes desta cultura. Dentre os resíduos, encontra-se o engaço, que pode ser utilizado como matéria prima na fabricação de materiais reciclados, por exemplo, o papel. O papel fabricado com fibra de bananeira pode ser empregado como material de embalagens na indústria de alimentos desde que algumas de suas características sejam conhecidas. Algumas destas características referem-se às propriedades térmicas, sendo as principais: calor específico, condutividade térmica e difusividade térmica. Em meio às metodologias aplicadas para a determinação destas propriedades, tem-se a termografia infravermelha, que é capaz de determinar a temperatura superficial de objetos através da coleta de imagens captadas por uma câmera que converte a radiação emitida por este objeto em sinais elétricos, criando uma imagem térmica. $O$ objetivo deste trabalho foi utilizar a termografia infravermelha na determinação das propriedades térmicas de materiais produzidos a partir de fibra de bananeira. Para tanto, observou-se a variação da temperatura superficial da amostra utilizando a termografia infravermelha. Foi implementado, em linguagem FORTRAN®, um algoritmo capaz de simular o decaimento da temperatura quando fornecidos valores da massa específica, calor específico, condutividade térmica e difusividade térmica do material e fluxo térmico imposto à amostra. Variaram-se os valores da condutividade térmica e calor específico, no modelo matemático, até o ajuste da curva numérica com a experimental, encontrando, respectivamente, $0,25 \mathrm{~W} /\left(\mathrm{m}^{2} . \mathrm{K}\right)$ e $1220 \mathrm{~J} /(\mathrm{kg} . \mathrm{K})$. A difusividade térmica foi calculada, indiretamente, sendo encontrado o valor de $6,44 \times 10^{-7} \mathrm{~m}^{2} / \mathrm{s}$. Conclui-se que a termografia infravermelha foi capaz de determinar as propriedades desde que alguns parâmetros sejam previamente determinados, como por exemplo, a emissividade da amostra. O modelo matemático foi fundamental como ferramenta de ajuste das curvas.

PALAVRAS-CHAVE: Fibra de bananeira. Termografia infravermelha. Calor especifico. Difusividade térmica. Condutividade térmica.

ABSTRACT: As one of the largest producers of bananas, Brazil is also a major generator of waste from this culture. Among the waste, the flowering stalk can be used as feedstock in the manufacture of recycled materials, for example, paper. The paper made from banana fiber can be used as packaging material in the food industry since some of its characteristics are known. Some of these features refer to the thermal properties, the major being the specific heat, thermal conductivity and thermal diffusivity. Amid the methodologies for determining these properties, it has infrared thermography, which is able to determine the surface temperature of objects by images captured by a camera that converts the radiation emitted by this object into electrical signals, creating a thermal image. The objective of this study was to use infrared thermography in the determination of thermal properties of materials 
made from banana fiber. For this, observed the variation of surface temperature of the sample using infrared thermography. It was implemented in FORTRAN $\AA$, an algorithm to simulate the decay of the temperature when given values of density, specific heat, thermal conductivity and thermal diffusivity of the material and heat flux imposed on the sample. Varying the values of thermal conductivity and specific heat in the mathematical model, to the numerical curve fitting with the experimental finding, respectively, $0.25 \mathrm{~W} /\left(\mathrm{m}^{2} . \mathrm{K}\right)$ and $1220 \mathrm{~J} /(\mathrm{kg} . \mathrm{K})$. The thermal diffusivity was calculated indirectly, and with values of $6.44 \times 10^{-7} \mathrm{~m}^{2} / \mathrm{s}$. It is concluded that infrared thermography was able to determine the properties since some parameters are predetermined, such as the emissivity of the sample. The mathematical model was key to the curves adjustment tool.

KEYWORDS: Fiber banana plant. linfrared thermography. Specific heat. Thermal diffusivity. Thermal conductivity.

\section{INTRODUÇÃO}

A banana (Musa spp.) é nativa do sudoeste da Ásia (NÓBREGA, 2006; OLIVEIRA, 2010) e apresenta uma grande importância socioeconômica para muitos países como Equador e Colômbia, dentre outros da América Latina (CARDOSO, 2005; OLIVEIRA, 2010).

O Brasil ocupa lugar de destaque no cenário mundial de cultivo de banana, sendo o quarto maior produtor mundial, ficando, apenas, atrás da Índia, China e Filipinas (Food and Agriculture Organization - FAO, 2011); tal posição coloca o país também como grande gerador de resíduos provenientes da colheita deste produto. Dentre os resíduos, têm-se as folhas, o pseudocaule e o engaço. As folhas e o pseudocaule são usados, comumente, no solo como cobertura morta, minimizando custos de adubação; já o engaço é descartado no momento de separação da penca, e geralmente, tratado como lixo (SOFFNER, 2001).

Visando atender uma demanda ambiental, seja pelo emprego de matérias primas de baixo custo ou pelo reaproveitamento de materiais descartados, o engaço da bananeira pode ser utilizado para a geração de energia e como matéria prima para a produção de polpa celulósica e papel (CHERTMAN \& SIMÕES-MOREIRA, 2008). O material celulósico proveniente do reaproveitamento do engaço pode ser usado, por exemplo, como embalagem secundária e/ou terciária na indústria de alimentos.
Para ser utilizado como embalagem, o material deve possuir determinadas características, entre elas, propriedades térmicas adequadas. Segundo Santos (2005), dentre as propriedades térmicas, as mais importantes são a condutividade térmica, o calor específico e a difusividade térmica, pois descrevem condições de resistência ao choque térmico e dissipação de calor mediante variações de temperaturas ambientais.

O conceito de condutividade térmica é oriundo da lei de Fourier, visualizado através da Eq. 1.

$\bar{q}_{x}=-k A \frac{\partial T}{\partial x}$

onde $\vec{q}_{x}$ é a taxa de calor transmitido por condução; $k$ é a condutividade térmica do material; $A$ é área da seção através da qual o calor fluirá, perpendicular à direção do fluxo de calor, $\partial T / \partial x$ é o gradiente de temperatura, com a distância $x$, na direção do fluxo de calor (MATUDA, 2008).

A condutividade térmica é uma propriedade importante para a determinação das taxas de aquecimento e resfriamento admissíveis e do perfil de temperatura resultante de alguma operação no material (AKIYOSHI, SILVA \& PANDOLFELLI, 2001).

Segundo Schmidt, Henderson \& Wolgemuth (1996), a condutividade térmica pode ser determinada a partir da análise da Equação geral da difusão do calor (Eq. 2).

$\frac{\partial}{\partial x}\left(\frac{\partial I}{\partial x}\right)+\frac{\partial}{\partial y}\left(\frac{\partial I}{\partial y}\right)+\frac{\partial}{\partial z}\left(k \frac{\partial T}{\partial z}\right)+\frac{\bar{q}}{k}=\frac{1}{\alpha} \frac{\partial I}{\partial t}$ 
onde $T$ é a temperatura em função das coordenadas $x, y$ e $z, t$ é o tempo e a é a difusividade térmica, definida como (Eq. 3):

$\alpha=\frac{k}{\rho c_{p}}$

Onde $k$ é a condutividade térmica, $\rho$ é a massa específica e $c_{p}$ é o calor específico do sólido analisado.

Uma das possibilidades para a determinação de propriedades térmicas é a termografia infravermelha, sendo esta, classificada como um ensaio não destrutivo que pode ser utilizado para determinar a temperatura superficial de objetos (NUNES et al., 2008; IBARRACASTANEDO et al., 2004). Câmeras apropriadas coletam a radiação infravermelha emitida pela superfície e a converte em sinais elétricos, criando imagens térmicas do campo de temperatura (BARREIRA \& FREITAS, 2007; NUNES et al., 2008). As imagens fornecidas pelas termocâmeras são chamadas de termogramas, que exibem as diferentes temperaturas de um determinado local da amostra na forma de gradientes de coloração (escala policromática) ou de tonalidades de cinza (escala monocromáticas), conforme mencionado por Tarpani et al. (2009). Segundo Tavares (2006), para que a termografia possa identificar a distribuição da temperatura superficial é necessário um diferencial de temperatura entre o corpo e o meio.

Segundo Maldague (2002), a termografia infravermelha pode ser classificada em ativa e passiva, de acordo com a excitação térmica utilizada.

Para a termografia passiva (Fig. 1), é necessário um diferencial natural de temperatura entre a amostra e o meio o qual se encontra, ou seja, nenhuma estimulação térmica artificial é utilizada (TAVARES, 2006). Já para a termografia ativa (Fig. 2), um estímulo externo é indispensável para induzir os contrates térmicos entre a amostra e o ambiente (MALDAGUE, 2002).

Dos métodos de excitação térmica, a termografia pulsada ou Pulsed Thermography (PT), é o mais tradicional (TAVARES, 2006). Consiste em um rápido aquecimento da amostra e, logo em seguida, o monitoramento do decaimento da temperatura (MELO, 2009). Além da ampla utilização da PT em indústria aeroespacial e na detecção de falhas em estruturas, Maldague (2000) cita o seu uso na determinação de propriedades térmicas de materiais.

Também chamada de lock-in, a termografia modulada ou modulated thermography (MT) consiste em submeter a amostra a uma estimulação térmica senoidal. Considerando o regime estacionário, a resposta térmica obtida por este estímulo também será senoidal, cuja amplitude e fase dependem da frequência de entrada (MALDAGUE \& MARINETTI, 1996).

Um pulso pode ser decomposto, matematicamente, em vários componentes senoidais individuais. $\mathrm{NaPT}$, ondas térmicas de diversas amplitudes e frequências são lançadas na amostra em regime transiente, enquanto que na MT uma onda térmica de frequência única é usada em regime estacionário. A possibilidade da associação destas duas técnicas foi relatada por Maldague, Galmiche \& Ziadi (2002), resultando na pulsed phase thermography (PPT), ou termografia pulsada por fase, em tradução livre.

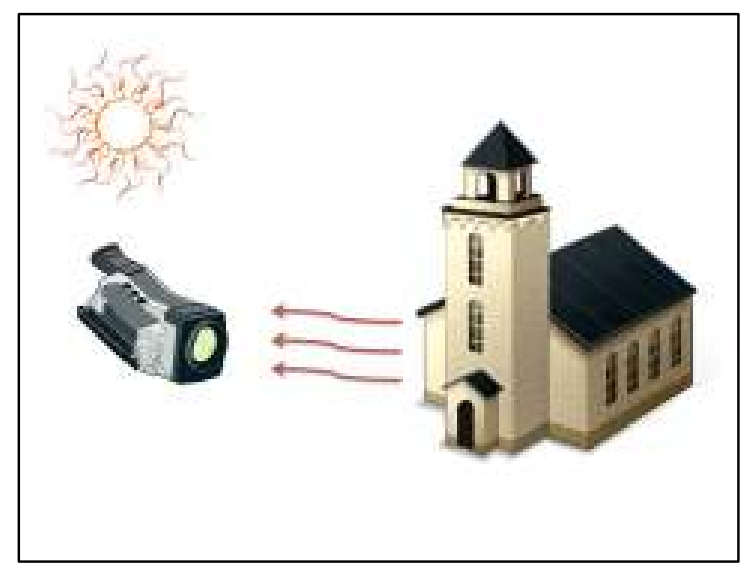

Figura 1 - Esquema de termografia passiva.

O objetivo deste trabalho foi utilizar a termografia infravermelha na determinação das propriedades térmicas de materiais produzidos a partir de fibras de bananeiras. 


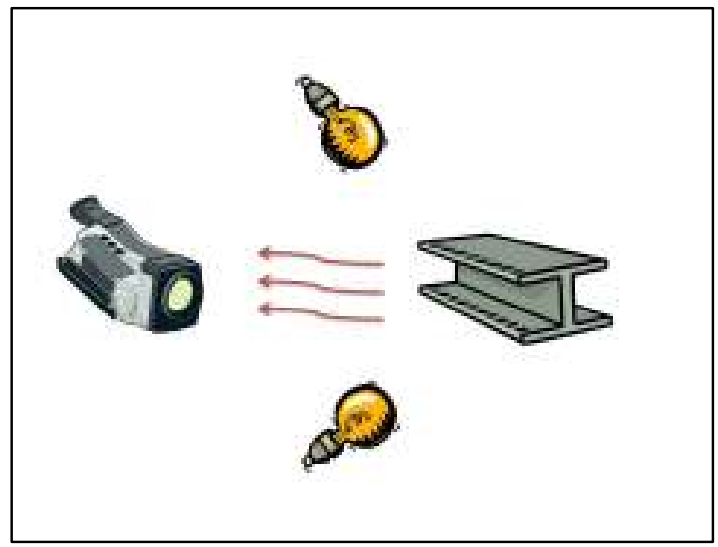

Figura 2 - Esquema de termografia ativa.

\section{MATERIAIS E MÉtOdOS}

Foi necessário estabelecer um critério para a realização das análises térmicas verificando a natureza do material, as condições ambientais, as características do equipamento, as fontes de incertezas e a facilidade de interpretação dos resultados. Por este lado, a termografia infravermelha apresenta grandes vantagens mesmo sendo necessária a montagem de uma bancada específica para este tipo de análise.

A seguir estão descritos o processo de produção do papel a partir da fibra de bananeira, a montagem da bancada de testes, a aplicação da termografia infravermelha na determinação das propriedades térmicas e o modelo matemático utilizado para validação dos resultados experimentais.

\subsection{PRODUÇÃO DO PAPEL}

A produção do papel foi realizada no Laboratório de Engenharia Química do Centro Universitário de Belo Horizonte UNI-BH.

Neste trabalho, foram utilizados $80 \%$ de papel para reaproveitamento e $20 \%$ de fibra vegetal. O papel foi recolhido nas próprias dependências do Centro Universitário de Belo Horizonte, selecionando materiais que possuíam poucas dobras e impressos preferencialmente em tinta preta. A fibra vegetal escolhida foi proveniente do engaço da bananeira, da cultivar nanicão. $O$ engaço foi recolhido no Ceasa Minas, localizado no município de Contagem - MG, e compreende desde o final do pseudocaule até o botão floral (coração).

Segundo a metodologia adaptada de Lacerda (2009), os papéis recolhidos foram picados em tiras com, aproximadamente, $1 \mathrm{~cm}$ de largura no sentido longitudinal. As tiras foram imersas em água e deixadas em repouso por $24 \mathrm{~h}$. Após este período, foram colocadas em um liquidificador convencional, na proporção de $100 \mathrm{~g}$ de papel para cada $1 \mathrm{~L}$ de água $\mathrm{e}$ processadas durante $3 \mathrm{~min}$. A base final foi reservada para posterior utilização.

O engaço da bananeira foi picado em pedaços pequenos e triturado em liquidificador convencional durante $5 \mathrm{~min}$. A fibra triturada foi lavada por três vezes com solução de hipoclorito de sódio a $2 \%$ de cloro ativo, na proporção de $50 \mathrm{~mL}$ da solução para cada litro de água. Conforme mencionado por Lacerda (2009), o uso do hipoclorito de sódio tem por finalidade o clareamento do papel a ser obtido.

Após as lavagens, a fibra foi adicionada em uma tina de inox, juntamente com a base preparada com o papel, na proporção de $20 \%$ e $80 \%$, respectivamente. Esta mistura foi cozida a $100^{\circ} \mathrm{C}$ por $120 \mathrm{~min}$, conforme sugerido por Soffner (2001).

Ao final do cozimento, a mistura ficou em repouso na própria tina até resfriamento à temperatura ambiente $\left(25^{\circ} \mathrm{C}\right)$, de forma natural. Já resfriada, foi triturada novamente em liquidificador convencional para melhor entrelaçamento das fibras, durante $3 \mathrm{~min}$. A seguir a mistura foi acondicionada em telas de $30 \mathrm{~cm} \times 40 \mathrm{~cm}$ e com orifícios de $1 \mathrm{~mm}$, onde ficaram em repouso por 24 h.

Decorrido esse tempo, as telas com a mistura foram levadas para uma estufa com convecção forçada de ar, marca TECNAL modelo TE 394/4, onde foram secas a 
$65^{\circ} \mathrm{C}$ por $60 \mathrm{~min}$. A velocidade do ar foi de $0,1 \mathrm{~m} / \mathrm{s}$ medida em anemômetro Marca Homis. A umidade do ar foi de 19,8\% medida em psicrômetro Marca Amprobe, modelo THWD-1. Quando secos, os papéis foram retirados das telas e encaminhados para análise térmica. O processo de produção do papel pode ser visualizado na Figura. 3.

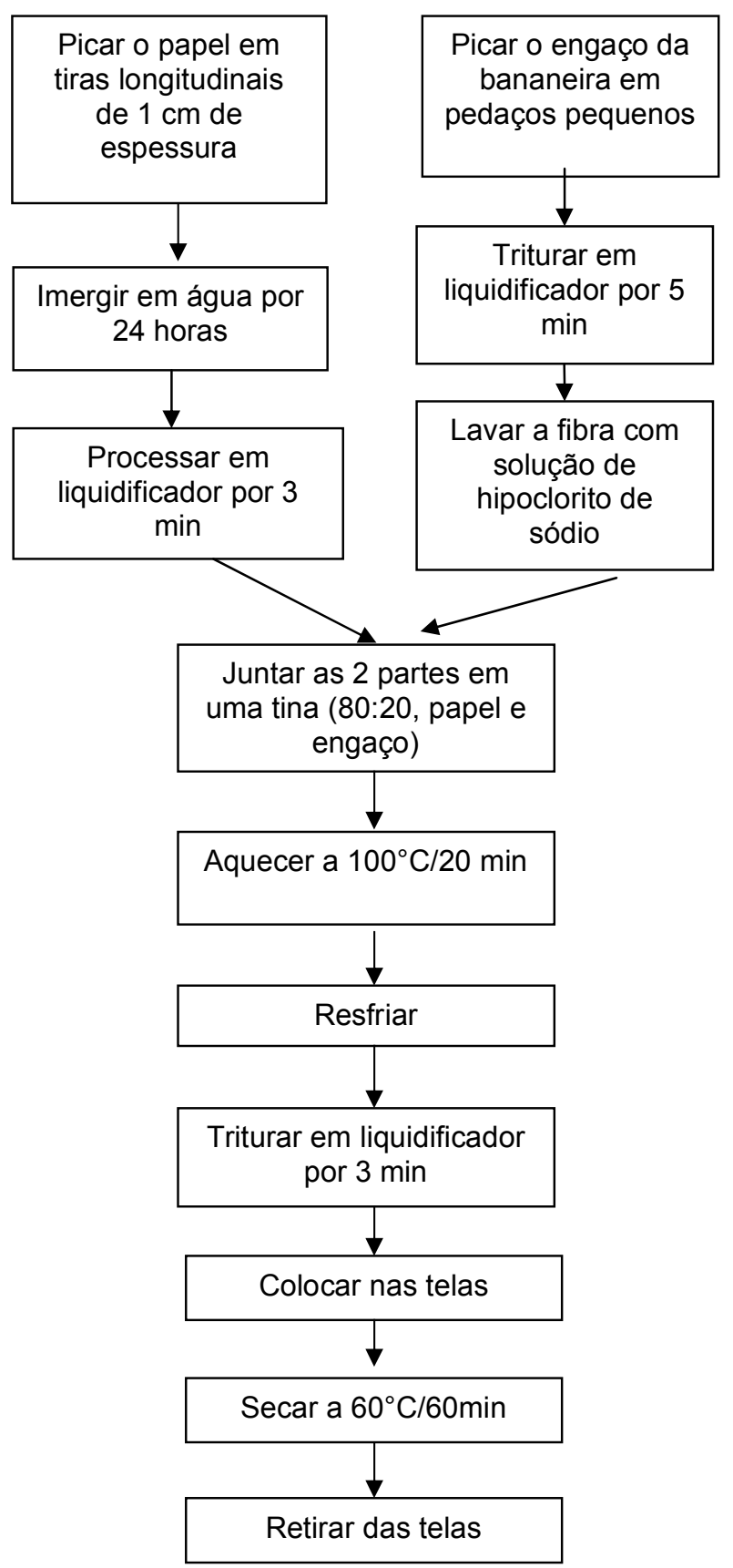

Figura 3 - Fluxograma de produção do papel.

\subsection{BANCADA DE TESTES}

A bancada de testes foi projetada e construída no Laboratório de Engenharia Química do Centro Universitário de Belo Horizonte - UNI BH e vem representada esquematicamente na Figura.4.

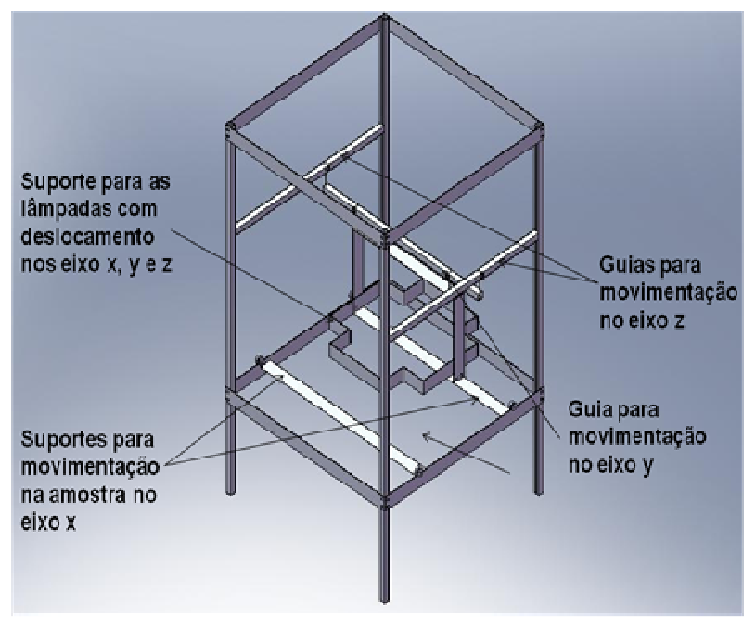

Figura 4: Esquema da bancada de testes.

As guias permitem a movimentação da amostra no eixo $y$ através de roldanas e no eixo $z$ por meio de roscas sem fim, localizadas na parte superior da bancada. Para movimentação no eixo x, a amostra deslizar, de acordo com o desejado, sobre o próprio suporte conectado à guia do eixo y. Assim a amostra tem movimentação estabelecida em todos os eixos.

O suporte para lâmpadas também permite movimentação do conjunto de lâmpadas nos três eixos cartesianos, podendo, assim, acompanhar todo o movimento da amostra. Este conjunto é composto por quatro lâmpadas halógenas, com potência de $1000 \mathrm{~W}$ cada, e por dispositivos de acionamento que permitem que todas acendam concomitantemente ou, então, em pares previamente estabelecidos.

\subsection{ENSAIOS TÉRMICOS NÃO DESTRUTIVOS PARA A DETERMINAÇÃO DAS PROPRIEDADES TÉRMICAS}

Antes de estabelecer o procedimento para determinar as propriedades térmicas do material, foi necessário 
identificar características inerentes ao meio ambiente e da própria amostra. Estas características são fundamentais para a identificação e controle das fontes de incerteza.

Para monitoramento da umidade do ar utilizou-se, psicômetro Marca Amprobe, modelo THWD-1. O psicrômetro foi posicionado ao lado da bancada de testes e ficou em repouso por 1 min para estabilização da indicação.

O valor fornecido foi anotado $e$ inserido, tanto na termocâmera, marca Flir Systems, modelo 160, durante os ensaios; quanto no programa ThermaCAM Researcher $2002^{\mathrm{TM}}$, fornecido pelo fabricante da termocâmera, durante a análise das imagens térmicas. Este procedimento foi repetido em cada uma das medições realizadas.

A distância entre a termocâmera e a amostra foi medida por meio de uma trena e o seu valor anotado e inserido como parâmetro na termocâmera e no programa ThermaCAM Researcher $2002^{\mathrm{TM}}$. Por padronização, estipulou-se a distância em $1 \mathrm{~m}$ em todas as medidas efetuadas, inclusive na determinação da temperatura refletida e emissividade do material.

O procedimento para determinação da temperatura refletida foi baseado no manual da termocâmera fornecido pela Flir Systems (2010).

Cortou-se um pedaço de folha de alumínio de aproximadamente $30 \times 40 \mathrm{~cm}$. Amassou-se esta porção e, a seguir, colou-se em um papel cartão do mesmo tamanho. O papel cartão com o alumínio foi posicionado no suporte da amostra, com o lado da folha de alumínio voltado para a termocâmera .

No painel da termocâmera, definiu-se a emissividade como sendo 1,0 e mediu-se o valor da temperatura aparente da folha de alumínio. Este valor foi anotado e entrou como referência quando solicitada a temperatura refletida durante a determinação da emissividade do material e realização dos ensaios térmicos.
Para a determinação da emissividade do material foi usada a técnica de termografia passiva. A amostra recebeu um pedaço de fita isolante $3 \mathrm{M}^{\mathrm{TM}}$ em sua superfície, de, aproximadamente, $6 \times 3 \mathrm{~cm}$, e foi posicionada recebendo diretamente a radiação proveniente dos raios solares. Foi mantida por 10 minutos nesta posição até estabilização da temperatura.

Com a termocâmera previamente ajustada, com o valor da temperatura refletida e a $1 \mathrm{~m}$ de distância e a $90^{\circ}$ da amostra, inseriu-se o valor da emissividade padrão da fita isolante, que é 0,97. Ajustou-se o foco da termocâmera na fita isolante, mediu-se sua temperatura e anotou-se o valor. Imediatamente, posicionou-se o foco na superfície da amostra e variou-se a emissividade até que a temperatura da amostra fosse condizente com a temperatura anotada da fita isolante. Anotou-se o valor da emissividade da amostra. O procedimento foi repetido por 12 vezes, adotando-se para a emissividade o valor médio das indicações obtidas.

$\mathrm{Na}$ determinação da massa específica da amostra utilizou-se uma balança experimental QUIMIS modelo BG 2000, onde se verificou a massa do material a ser estudado. Para as medidas de comprimento usou-se um escalímetro e para espessura, um paquímetro digital DIGIMESS. A média de 12 indicações de cada parâmetro foi obtida e utilizada na Eq. 4 para determinação da massa específica $\rho$.

$\rho=\frac{m}{w l \xi}$

onde $m$ é a massa média, $w$ é a largura média, $l$ é o comprimento médio e $\xi$ é a espessura média.

Para a determinação da taxa de calor recebido pela amostra utilizou-se, como padrão, um bloco de papel branco com emissividade e calor específico conhecidos. Segundo Incropera et al. (2008), o calor específico do papel branco é $1340 \mathrm{~J} /(\mathrm{kg} . \mathrm{K})$ e sua massa específica é $930 \mathrm{~kg} / \mathrm{m}^{3}$. De acordo com dados da Flir Systems 
(2010), a emissividade do papel varia de 0,7 a 0,9 . Adotou-se o valor médio de 0,8 como referências.

A folha de papel foi colocada no suporte de amostra da bancada de testes. Ajustou-se o foco da termocâmera, posicionada a $1 \mathrm{~m}$ do material, e inseriu-se o valor de 0,8 para a emissividade. Acionou-se a termocâmera para captação da imagem e, imediatamente, ligou-se o conjunto das 4 lâmpadas da bancada situado a $50 \mathrm{~cm}$ da amostra. Após $15 \mathrm{~s}$ o conjunto de lâmpadas foi desligado e uma nova imagem térmica foi gerada.

O procedimento foi repetido por 12 vezes, respeitando um tempo de 5 min entre cada repetição com o objetivo de provocar um resfriamento natural do bloco de papel. As imagens captadas foram tratadas no programa ThermaCAM Researcher $2002^{\mathrm{TM}}$, inserindo os valores da temperatura ambiente e umidade relativa do ar presentes durante os ensaios. A temperatura média de cada imagem, no início e no final dos testes, foi registrada, sendo realizada, por fim, a média das 12 medições.

Para a determinação do calor, utilizou-se a Eq. 5:

$Q=m_{p} \Delta T$

onde $Q$ é o calor total emitido pelas lâmpadas após $15 \mathrm{~s}$. A taxa de calor então é encontrada dividindo o resultado pelo tempo.

Por se tratar de uma amostra que pode receber calor e, conseqüentemente, variar de temperatura em uma determinada faixa, a estimulação térmica foi realizada através de métodos artificiais (termografia ativa). A termografia ativa permite uma análise quantitativa das condições térmicas decorrentes da variação provocada pelos pulsos de energia; desde que a energia térmica aplicada seja devidamente conhecida, justificando a determinação da taxa de calor recebida pela amostra.

Após a excitação, nos ensaios utilizando a termografia pulsada ( $P T)$, imagens térmicas foram registradas em intervalos de tempo, sendo assim observado o decaimento temporal da temperatura.
A amostra foi posicionada no suporte localizado na bancada de testes e o conjunto de lâmpadas foi regulado a $50 \mathrm{~cm}$ da amostra, em posição centralizada ao suporte. A termocâmera teve seu foco ajustado, a distância e a angulação reguladas para $1 \mathrm{~m}$ e a $90^{\circ} \mathrm{em}$ relação à amostra, respectivamente. Inseriu-se no equipamento os valores de emissividade e temperatura refletida, que foram respectivamente, 0,8 e $26^{\circ} \mathrm{C}$. Estes valores foram constantes para todas as medições.

Acionou-se, concomitantemente, as 4 lâmpadas do conjunto, sendo mantidas em funcionamento durante 15 s. Imediatamente, desligou-se o conjunto de lâmpadas e registrou-se a primeira imagem térmica da amostra. Mais nove imagens térmicas foram registradas em intervalos regulares de $3 \mathrm{~s}$.

Todo o procedimento foi repetido por 12 vezes, respeitando um intervalo de 5 min entre cada ensaio para provocar o resfriamento natural da amostra. Foram anotadas, em cada caso, a temperatura ambiente e umidade relativa do ar.

As imagens térmicas obtidas foram tratadas no programa ThermaCAM Researcher $2002^{\mathrm{TM}}$, ajustando a temperatura ambiente e a umidade relativa do ar para cada ensaio, assim como a distância, emissividade e temperatura refletida. Estes últimos parâmetros foram considerados constantes em todos os ensaios.

\subsection{MOdelo MATEMÁTICO}

Para a determinação das propriedades térmicas do papel, foi utilizado modelo matemático baseado na solução numérica da Equação da difusividade térmica em coordenadas cartesianas e sem geração de calor (Eq. 6):

$\frac{\partial}{\partial x}\left(k \frac{\partial T}{\partial x}\right)+\frac{\partial}{\partial y}\left(k \frac{\partial T}{\partial y}\right)+\frac{\partial}{\partial z}\left(k \frac{\partial T}{\partial z}\right)=p c_{p} \frac{\partial T}{\partial t}$

onde $k$ é a condutividade térmica; $T$ é a temperatura em função das coordenadas $x, y$ e $z, t$ é o tempo; $\rho$ é a massa específica e $c_{p}$ é o calor específico. Como 
condições iniciais da análise térmica foram empregadas as Eq. 7 e 8:

$$
\begin{aligned}
& T_{1}((x, y, z, 0))=q /\left(\rho c_{1} p \xi\right), \text { para } 0<z<\mathrm{C} \\
& T_{1}((x, y, z, 0))=T_{1} 0, \text { paraz }>C
\end{aligned}
$$

onde $T_{0}$ é a temperatura inicial da amostra, ou seja, é a temperatura da amostra antes da excitação térmica; $\xi$ é a espessura mínima a ser considerada da amostra, isto é, a camada superficial que recebe e absorve o impulso térmico e $Q$ é o calor fornecido pelo conjunto de lâmpadas.

A amostra foi isolada adiabaticamente nas laterais e na superfície inferior, tendo-se, assim, um fluxo de calor unidimensional na direção z. Didaticamente, o comprimento da amostra é representado pelo eixo $\mathrm{x}$, sua largura pelo eixo y e sua espessura pelo eixo $z$, conforme Figura. 5.

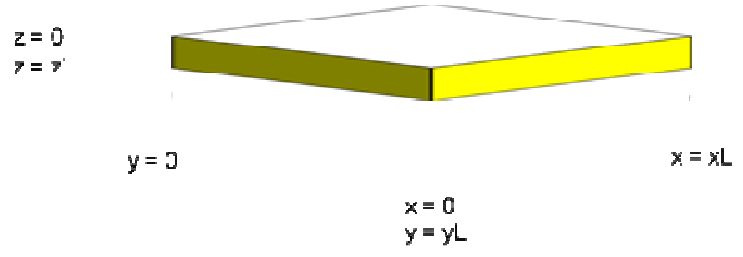

Figura 5 - Esquematização didática das coordenadas na amostra.

Assim, têm-se condições de contorno adiabáticas em $x=0, x=x L, y=0, y=y L$ e $z=z L$ e condições convectivas em $z=0$, conforme demonstrado nas Eq. 9 a 14:

$\left.k \frac{\partial T}{\partial x}\right|_{x=0}=0$

$-\left.k \frac{\partial T}{\partial x}\right|_{s=x L}=0$

$\left.k \frac{\partial T}{\partial y}\right|_{y=0}=0$

$-\left.k \frac{\partial T}{\partial y}\right|_{y=w L}=0$

$-\left.k \frac{\partial T}{\partial z}\right|_{y=n L}=\mathbf{0}$ $\left.k \frac{\partial T}{\partial z}\right|_{z=0}=\boldsymbol{h}\left(T-T_{\infty}\right)$

O sinal negativo representado nas Eq. 10, 12 e 13 representa que o fluxo de calor está em sentido contrário ao das Eq. 9 e 11. O coeficiente convectivo $h$ adotado foi de $12 \mathrm{~W} /\left(\mathrm{m}^{2} . \mathrm{K}\right)$ Conforme estipulado por Incropera et al. (2008), tomando como base a média de valores para convecção livre de gases. A temperatura ambiente, $T_{\infty}$, foi obtida experimentalmente.

Segundo Grossi (2008), para solução numérica da difusão do calor pode-se utilizar o método dos volumes finitos. O método de volumes finitos é utilizado para obter as equações aproximadas, satisfazendo a conservação da propriedade em nível de volumes elementares (MALISKA, 2004).

Pela integração da Eq. 6, no espaço e no tempo, pela aproximação das derivadas nas interfaces do volume elementar por diferenças centrais e usando uma formulação totalmente implícita no intervalo temporal ( $T_{0}$ no tempo presente e $\mathrm{T}$ no tempo futuro), tem-se, segundo Maliska (2004) e Patankar (1991):

$A_{p} T_{P}=A_{g} T_{E}+A_{W} T_{W}+A_{q} T_{W}+A_{s} T_{S}+A_{f} T_{F}+A_{b} T_{E}$ (15)

$A_{p}=\frac{k}{c_{p} \Delta x} \mid \Delta y \Delta z$

$A_{w}=\left.\frac{k}{c_{p} \Delta x}\right|_{w} \Delta y \Delta z$

$A_{n}=\left.\frac{k}{c_{p} \Delta y}\right|_{n} \Delta x \Delta z$

$A_{s}=\left.\frac{k}{\sigma_{p} \Delta y}\right|_{s} \Delta x \Delta z$

$A_{j}=\frac{k}{c_{p} \Delta \underline{z}} \mid \Delta x \Delta y$

$A_{p}=c_{c_{p} \Delta z}^{k} \Delta x \Delta y$

$B_{F}=A_{P}^{0} T_{P}^{0}$

$A_{p}^{0}=\frac{\rho \Delta x \Delta y \Delta z}{\Delta t}$ 
$A_{p}=A_{e}+A_{w}+A_{n}+A_{s}+A_{f}+A_{b}+A_{P}^{0}$

Para a solução da Eq. 15, foi desenvolvido um algoritmo em linguagem FORTRAN®, o qual fornece a temperatura em cada ponto selecionado dentro do volume de controle, conforme sugestão de Patankar (1991) e aplicação de Tavares (2006).

\section{RESULTADOS E DISCUSSÃO}

\subsection{DETERMINAÇÃO DOS PARÂMETROS ESSENCIAIS PARA O AJUSTE DAS IMAGENS TÉRMICAS}

$\mathrm{Na}$ avaliação da umidade foi observada pouca variação durante a determinação da temperatura refletida (Figura. 6) e no decorrer das repetições dos ensaios térmicos. Esta pequena variação pode ser explicada devido ao calor emitido pelo conjunto de lâmpadas, que ocasionou um decaimento de umidade.

Estes resultados demonstram a importância do conhecimento do valor de umidade do ar para cada imagem térmica, já que este parâmetro influencia no resultado da medição de temperatura pela termocâmera (FLIR SYSTEMS, 2010).

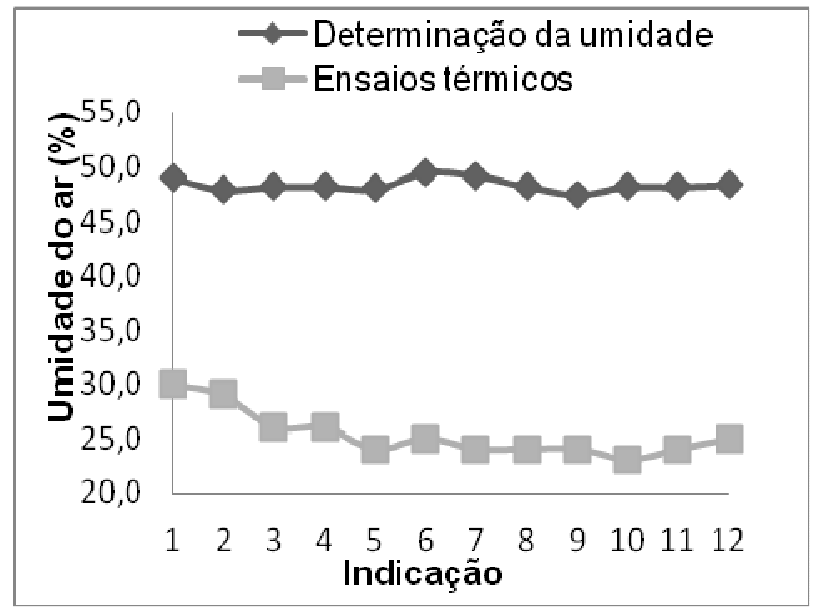

Figura 6 - Umidade do ar.
Das 12 imagens térmicas realizadas da folha de alumínio, 1 foi descartada por apresentar-se desfocada. Pela TAB. 1 pode-se verificar a temperatura refletida em cada indicação, acompanhada de sua respectiva temperatura ambiente. Como valor de referência foi adotada a média destes valores, $25,5^{\circ} \mathrm{C}$, sendo então inserido na termocâmera para os subseqüentes ensaios térmicos.

Não há diferença significativa $(p<0,05)$ entre a média da temperatura refletida e a média da temperatura ambiente. Assim, nas condições de laboratório aplicadas, a própria temperatura ambiente pode ser empregada como temperatura refletida, conforme foi utilizado por Tavares (2006).

Tabela 1 - Temperaturas refletidas e suas respectivas temperaturas ambiente.

\begin{tabular}{ccc}
\hline Indicação & $\begin{array}{c}\text { Temperatura } \\
\text { refletida } \\
\left({ }^{\circ} \mathbf{C}\right)\end{array}$ & $\begin{array}{c}\text { Temperatura } \\
\text { ambiente }\left({ }^{\circ} \mathrm{C}\right)\end{array}$ \\
\hline 1 & 25,4 & 25,7 \\
2 & 25,4 & 25,7 \\
3 & 25,4 & 25,7 \\
4 & 25,6 & 25,8 \\
5 & 25,5 & 25,8 \\
6 & 25,5 & 25,8 \\
7 & 25,8 & 25,8 \\
8 & Desfocada & Desfocada \\
9 & 25,6 & 25,8 \\
10 & 25,6 & 25,8 \\
11 & 25,7 & 25,9 \\
12 & 25,3 & 25,7 \\
\hline Média & 25,8 & 25,5 \\
Desvio Padrão & 0,1 & 0,1 \\
\hline
\end{tabular}

Por se tratar de um material novo, não há padrões de referências na literatura para a emissividade. Avdelidis \& Moropoulou (2003) também utilizaram a termografia infravermelha para determinar a emissividade de novos materiais e sugeriram a comparação com estruturas semelhantes; assim, tomando como base valores de papel branco, observa-se que a emissividade da amostra é bem menor. Enquanto o papel branco apresenta valores de 0,7 a 0,9 (FLIR SYSTEMS, 2010) ou 0,92 a 0,97 (INCROPERA et al., 2008), a amostra 
apresentou valor médio de 0,39. Uma das justificativas para tal discrepância é em relação à composição do material, já que, além de papel reciclado também apresenta fibra de bananeira. Outro ponto é referente à rugosidade e coloração mais escura do papel com fibra.

Encontrou-se o valor médio de $318,12 \mathrm{~kg} / \mathrm{m}^{3}$ como massa específica. Novamente, por falta de dados de literatura por se tratar de um material novo, tomou-se como referência o papel branco, que apresenta 930 $\mathrm{kg} / \mathrm{m} 3$ (INCROPERA et al., 2008). Pode-se justificar o valor mais baixo para a amostra devido ao processo de obtenção deste papel que, por ser de forma artesanal, não permite uma eficiente compactação durante a etapa de prensagem.

$\mathrm{Na}$ TAB. 2 estão representadas as temperaturas da folha de papel branco antes $(t=0 \mathrm{~s})$ e depois $(t=15 \mathrm{~s})$ da excitação térmica proveniente das lâmpadas da bancada, durante as 12 repetições realizadas.

Tabela 2 - Temperaturas antes e depois da excitação térmica

\begin{tabular}{ccc}
\hline Indicação & $\begin{array}{c}\text { Temperatura } \\
\left({ }^{\circ} \mathbf{C}\right) \\
(\mathbf{t =} \mathbf{0} \mathbf{~})\end{array}$ & $\begin{array}{c}\text { Temperatura } \\
\left({ }^{\circ} \mathbf{C}\right) \\
(\mathbf{t}=\mathbf{1 5} \mathbf{~ s})\end{array}$ \\
\hline 1 & 27,1 & 63,2 \\
2 & 27,4 & 64,3 \\
3 & 27,3 & 64,0 \\
4 & 28,6 & 65,0 \\
5 & 26,1 & 61,1 \\
6 & 26,5 & 61,4 \\
7 & 27,1 & 62,8 \\
8 & 26,9 & 62,5 \\
9 & 27,0 & 62,9 \\
10 & 27,3 & 62,9 \\
11 & 26,9 & 62,0 \\
12 & 27,0 & 62,4 \\
\hline Média & 27,1 & 62,9 \\
Desvio Padrão & 0,6 & 1,1 \\
\hline
\end{tabular}

Utilizando a diferença das médias de temperatura, a média da massa que foi de 49,99 g e o valor de literatura do calor específico para o papel branco, têmse, pela Eq. 7, aproximadamente $2396 \mathrm{~J}$ de calor recebido pelo papel durante os $15 \mathrm{~s}$ de excitação térmica. Dividindo este resultado pelo tempo da excitação, chegou-se à taxa de calor de aproximadamente $160 \mathrm{~W}$.

\subsection{ENSAIOS TÉRMICOS}

Cada uma das 12 indicações apresentou 10 imagens térmicas que foram tratadas computacionalmente. Para ilustração, uma das indicações está representada pela Figura. 7 , onde se observa a variação da temperatura por intermédio de sua respectiva escala colorimétrica. Nos itens (1), (2) e (3) da mesma figura, existem zonas de coloração diferentes no interior das imagens, provavelmente associadas às falhas do material. Tavares (2006) e Tavares et al. (2006) utilizaram a termografia para identificar justamente falhas internas em obras de artes. Já Mendonça (2005) encontrou patologias no concreto com o auxílio de termocâmeras.
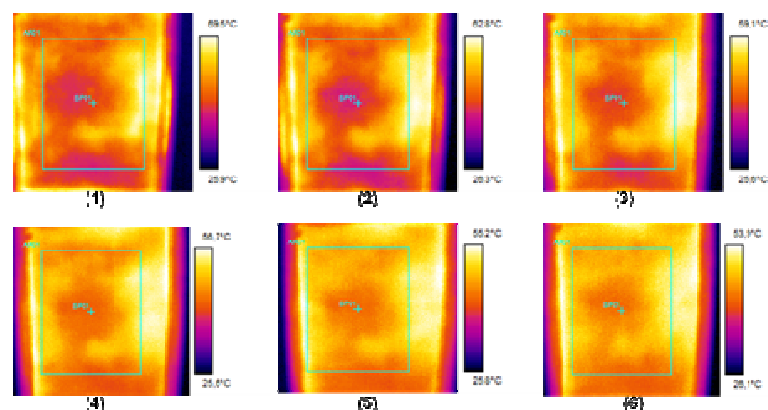

(5)
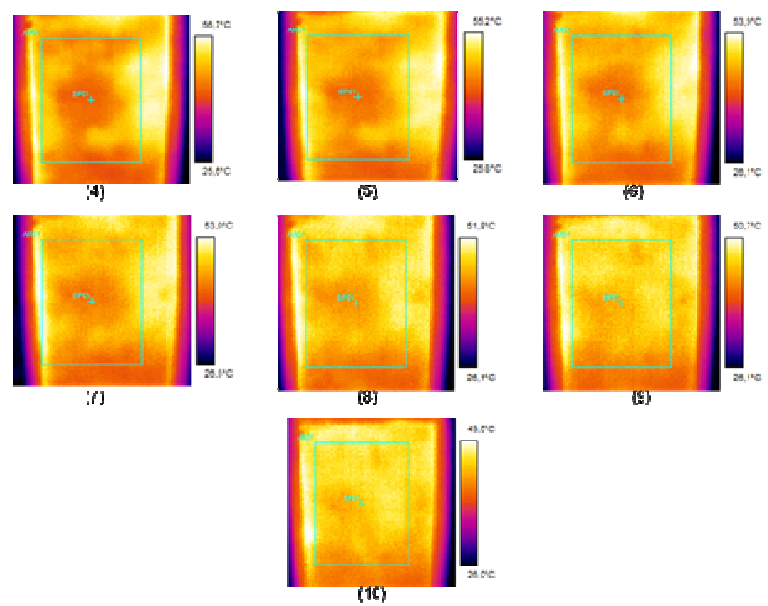

Figura 7 - Imagens térmicas tratadas do decaimento de temperatura da amostra: (1) em t $=0 \mathrm{~s} ;(2)$ em $\mathrm{t}=3 \mathrm{~s}$; (3) em t = 6; (4) em t = 9 s; (5) em t = $12 \mathrm{~s}$; (6) em t $=15$ $\mathrm{s} ;(7)$ em t $=18 \mathrm{~s} ;(8)$ em t $=21 \mathrm{~s}$; (9) em t $=24 \mathrm{~s} \mathrm{e} \mathrm{(10)}$ $\mathrm{em} \mathrm{t}=27 \mathrm{~s}$.

Para identificação das propriedades térmicas, utilizou-se o programa desenvolvido em ambiente FORTRAN®, onde foram introduzidos os valores determinados 
experimentalmente para a massa específica, temperatura ambiente, calor recebido e espessura da amostra.

Os valores da condutividade térmica e calor específico foram variados até que as temperaturas obtidas no modelo matemático apresentassem diferenças de, no máximo $1^{\circ} \mathrm{C}$, em relação à média das temperaturas obtidas experimentalmente. Esta diferença foi utilizada por representar a incerteza padrão encontrada na literatura (MAGNANI \& SILVA, 2007) para esta termocâmera.

A Figura. 8 apresenta o resultado comparativo entres as temperaturas obtidas experimentalmente e pelo modelo matemático.

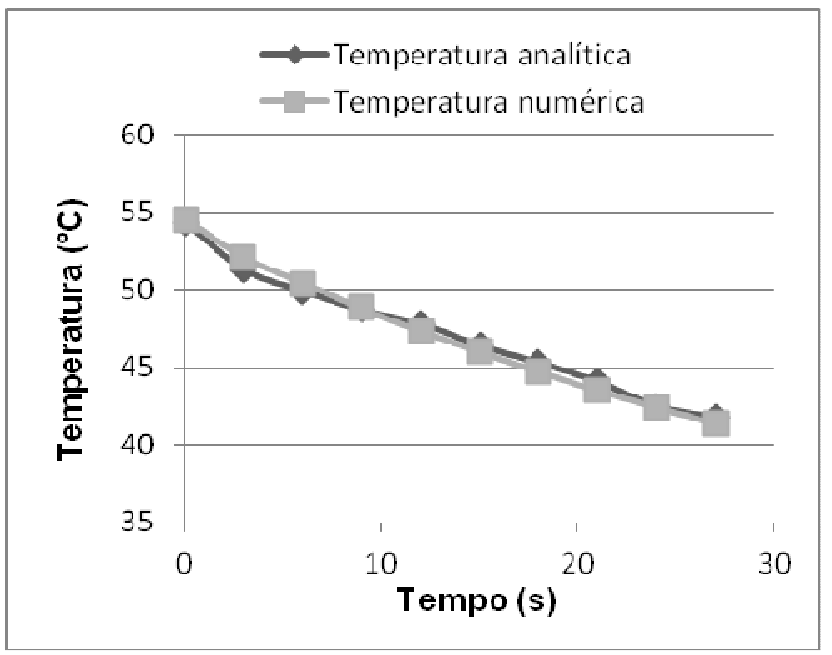

Figura 8 - Decaimento da temperatura (experimental e numérica).

A utilização do modelo matemático permitiu a determinação da condutividade térmica e calor específico das amostras como sendo, respectivamente, 0,25 W/(m.K) e $1220 \mathrm{~J} /(\mathrm{kg} . \mathrm{K})$. Dados próximos são citados por Incropera et al. (2008) para papel branco, o qual cita $0,18 \mathrm{~W} /(\mathrm{m} . \mathrm{K})$ para condutividade e 1340 $\mathrm{J} /(\mathrm{kg} . \mathrm{K})$ para o calor específico.

Já na determinação da difusividade térmica, utilizou-se a Eq. 4, substituindo os valores encontrados para a amostra do calor específico, condutividade térmica e massa específica. Obteve-se um valor de $\alpha$ de 6,44 $\mathrm{x}$ $10^{-7} \mathrm{~m}^{2} / \mathrm{s}$, enquanto que pelos dados da literatura (INCROPERA, 2008), a difusividade de um papel branco seria de $1,44 \times 10^{-7} \mathrm{~m}^{2} / \mathrm{s}$. Tal diferença foi provocada principalmente pela menor massa específica do papel fabricado com fibra de bananeira.

\section{ConClusões}

Durante a fabricação do papel, prensagem foi realizada de forma natural, não permitindo a completa compactação da base, gerando uma massa específica muito inferior ao padrão considerado (papel branco). A massa específica pode influenciar nas demais propriedades do material.

A metodologia utilizada exigiu que alguns parâmetros fossem previamente identificados, como a massa específica e a emissividade da amostra bem como a taxa de calor emitida pelo conjunto de lâmpadas da bancada e fatores extrínsecos, como temperatura ambiente, temperatura refletida e umidade relativa do ar. Assim, procedimentos para estas identificações foram empregados e podem servir como referência em análises futuras.

A umidade relativa do ar mostrou-se variável durante a execução dos ensaios, apresentando um decréscimo quando o conjunto de lâmpadas foi acionado. Por tratarse de um dado de entrada na termocâmera, deve ser constantemente monitorado. Já a temperatura refletida não apresentou uma diferença significativa quando comparada com a temperatura ambiente, ou seja, nas condições de laboratório utilizadas, não é necessário proceder a técnica de avaliação descrita neste trabalho, somente o controle da temperatura ambiente durante os ensaios térmicos.

Em relação à determinação das propriedades térmicas, o método da termografia infravermelha permitiu quantificar, como o auxílio do modelo matemático, o calor específico, condutividade e difusividade térmicas. Por tratar-se de um material novo e sem referência de 
literatura, adotou-se o papel branco como padrão mais próximo de comparação. Obtiveram-se valores próximos a este padrão, exceto em relação à emissividade, que neste estudo foi obtida experimentalmente e à difusividade térmica. A discrepância encontrada pode ser justificada muito provavelmente pela adição da fibra de bananeira quer possui propriedades distintas do papel.

O modelo matemático utilizado foi de fundamental importância na determinação das propriedades citadas e sua implementação permitiu comparar o decaimento experimental da temperatura com 0 decaimento numérico e assim ajustar as curvas até resolução dos valores das propriedades térmicas.

Para melhor confiabilidade é necessário realizar a avaliação das incertezas de medição de cada uma das etapas metodológicas a fim de garantir resultados de medição mais seguros.

\section{REFERÊNCIAS}

AKIYOSHI, M. M.; SILVA, A. P. da; PANDOLFELLI, V. C. Condutividade térmica e sua correlação com a temperatura e a massa específica volumétrica de materiais refratários sílico-aluminosos e aluminosos. Cerâmica, São Paulo, v. 47, n. 301, p. 19-27, jan./fev./mar., 2001. Disponível em: http://www.scielo.br/pdf/\%0D/ce/v47n301/a0547301.pd f. Acesso em: 25 mar. 2011.

AVDELIDIS, N. P.; MOROPOULOU, A. Emissivity considerations in building thermography. Energy and Buildings, Amsterdã, v. 35, p. 663-667, ago., 2003. Disponível em: http://www.sciencedirect.com/science/article/pii/S0378 778802002104 Acesso em: 21 mar. 2011.

BARREIRA, E.; FREITAS, V. P. de. Evaluation of building materials using infrared thermography. Construction and Building Materials, Amsterdã, v. 21, p. 218-224, jan., 2007. Disponível em: http://www.sciencedirect.com/science? ob=ArticleURL \& udi=B6V2G-4GY871G-

$8 \&$ user $=10 \&$ coverDate $=01 \% 2 \mathrm{~F} 31 \% 2 \mathrm{~F} 2007 \&$ rdoc $=$ $1 \&$ fmt $=$ high \& orig $=$ gate

way\& origin $=$ gateway $\&$ sort $=\mathrm{d} \&$ docanchor $=\&$ view $=\mathrm{C}$ \& searchStrld $=1756667156 \&$ rerunOrigin $=$ google \& a cct $=$ C000050221\& version $=1 \&$ urlVersion $=0$ \& userid $=10 \& \mathrm{md} 5=a 72 \mathrm{fe} 1 \mathrm{cab} 3 \mathrm{fc} 7929 \mathrm{a} 0 \mathrm{~b} 45 \mathrm{~d} 92 \mathrm{ccdb} 0 \mathrm{ba}$ \&sea $\underline{\text { rchtype }}=a$. Acesso em: 18 maio 2011.

CARDOSO, R. M. de C. B. Avaliação quantitativa de perdas pós-colheita de banana comercializada na cidade de Santo Antônio de Jesus - Bahia. 2005. 120 f. Dissertação (Mestrado em Ciências Agrárias) Escola de Agronomia, Universidade Federal da Bahia. Cruz das Almas, 2005. Disponivel em: http://www.bibliotecadigital.ufba.br/tde busca/arquivo .php?codArquivo=133. Acesso em: 27 mar. 2011.
CHERTMAN, M.; SIMÕES-MOREIRA, J. R. Secagem de papel produzido a partir do pseudocaule de bananeira. Boletim Técnico da Escola Politécnica da USP, São Paulo, n. 0811, 14 p., 15 maio 2008. Disponível em: http://www.mecanicapoliusp.org.br/05pesq/cont/pdf/811.pdf. Acesso em: 21 mar. 2011.

FLIR SYSTEMS $®$. Manual do operador: FLIR bxx series, FLIR ixx series. Canada: FLIR Systems, Inc. 2010.

FOOD AND AGRICULTURE ORGANIZATION (FAO). FAOSTAT. Roma, 2011. Disponível em: http://faostat.fao.org/site/339/default.aspx. Acesso em: 21 mar. 2011.

IBARRA-CASTANEDO, C.; GONZÁLEZ, D.; KLEIN, M.; VALLERAND, S.; MALDAGUE, X. Infrared image processing and data analysis. Infrared Physics \& Technology, Amsterdã, v. 46, p. 75-83, 2004. Disponível em: http://www.sciencedirect.com/science larticle/pii/S1350449504000520. Acesso em: 21 mar. 2011.

INCROPERA, F. P.; DEWITT, D. P.; BERGMAN, T. L.; LAVINE, A. S. Fundamentos de transferência de calor e de massa. Tradução de Eduardo Mach Queiroz e Fernando Luiz Pellegrini Pessoa. 6 ed. Rio de Janeiro: LTC, 2008. xix, 643 p. Título original: Fundamentals of heat and mass transfer.

LACERDA, N. O. S. Produção de papel artesanal de fibra de bananeira: uma proposta de ensino de química por projeto. 2009. 253 f. Dissertação (Mestrado Profissional em Ensino de Ciências) Instituto de Ciências Biológicas, Universidade de 
Brasília. Brasília, 2009. Disponível em: http://egroups.unb.br/ppgec/dissertacoes/2009/trabalhos/diss ertacao nilia lacerda.pdf. Acesso em: 21 mar. 2011.

MAGNANI, F. S.; SILVA, R. N. T. da. Infrared thermography applied to the quantitative determination of spatial and thermophysical parameters of hidden included objects. Applied Thermal Engineering, Amsterdã, v. 27, p. 2378-2384, 2007. Disponível em: http://www.sciencedirect.com/science/article/pii/S1359 431107000920. Acesso em: 18 mar. 2011.

MALDAGUE, X. Applications of infrared thermography in nondestructive evaluation. Trends in optical nondestructive testing and inspection (invited chapter), Pramod Rastogi ed., p. 591-609, 2000. Disponível em: http://w3.gel.ulaval.ca/ maldagx/r 1123.pdf. Acesso em: 02 abr. 2011.

MALDAGUE, X. GALMICHE, F.; ZIADI, A. Advances in pulsed phase thermography. Infrared Physics \& Technology, Maryland, v. 43, p. 174-181, 2002. Disponível em: http://citeseerx.ist.psu.edu/viewdoc/download?doi=10. 1.1.9.1197\&rep=rep1\&type=pdf. Acesso em: $17 \mathrm{mar}$. 2011.

MALDAGUE, $X$. Introduction to NDT by active infrared thermography. Materials Evaluation, Columbus, v. 6, p. 1060-1073, 2002. Disponível em: www.gel.ulaval.ca/ maldagx/ r 1221t.pdf. Acesso em: 02 abr. 2011.

MALDAGUE, X.; MARINETTI, S. Pulse phase infrared thermography. Journal Applied Physics., Nova lorque, v. $79, \quad$ p. $2694-2698,1996$. Disponível em: http://citeseerx.ist.psu.edu/viewdoc/download?doi=10. 1.1.33.1132\&rep=rep1\&type=pdf. Acesso em: $17 \mathrm{mar}$. 2011.

MALISKA, C. R.. Transferência de calor e mecânica dos fluidos computacional. 2. ed. rev. e ampl. Rio de Janeiro: LTC, 1995. $453 \mathrm{p}$.

MATUDA, T. G. Estudo do congelamento da massa de pão: determinação experimental das propriedades termofísicas e desempenho de panificação. 2008. 153 f. Tese (Doutorado em Engenharia) - Escola Politécnica, Universidade de São Paulo, São Paulo, $2008 . \quad$ Disponível em: http://www.teses.usp.br/teses/disponiveis/3/3137/tde17112008-114317/pt-br.php. Acesso em: 23 mar. 2011.

MELO, A. G. B. de. Inspeção de juntas coladas de tubos feitos por compósitos por termografia ativa pulsada. 2009. 63 f. Dissertação (Mestrado em Engenharia, Modalidade Profissional, Especialidade Engenharia de Inspeção de Equipamentos) - Escola de Engenharia, Universidade Federal do Rio Grande do Sul, Porto Alegre, 2009. Disponível em: http://www.lume.ufrgs.br/bitstream/handle/10183/1843 0/000728628.pdf?sequence=1. Acesso em: 26 mar. 2011.

MENDONÇA, L. V. Termografia por infravermelhos Inspeção de betão. Revista Engenharia \& Vida, Lisboa, n. 16, p. 53-57, set. 2005. Disponível em: http://www.spybuilding.com/downloads/termografia.pdf . Acesso em: 2 abr. 2011.

NÓBREGA, J. P. R. Produção de mudas de bananeira (Musa sp. AAB) em função da poda e doses de nitrogênio e boro. 2006. 97 f. Dissertação (Mestrado em Agronomia) - Departamento de Fitotecnia, Centro de Ciências Agrárias, Universidade Federal da Paraíba, Areias, 2006. Disponível em http://www.cca.ufpb.br/ppga/pdf/mestrado/Jose-piresms06.pdf. Acesso em: 17 maio 2011.

NUNES, J.; DOMINGUES, C.; DINHO, P.; PITARMA, R. Avaliação termográfica de instalações de refrigeração. In: CONGRESSO LUSOMOÇAMBICANO DE ENGENHARIA, 5. 2008, Maputo. Anais... Porto: Edições INEGI, 2008. p. 1-6. Disponível em:

http://www.cmade.ubi.pt/pdf/a international conferences/TIV refri gera\%C3\%A7\%C3\%A30.pdf. Acesso em: 17 mar. 2011.

OLIVEIRA, H. S. de. Comportamento de cultivares de bananeira (Musa spp) resistentes a doenças no processo de micropropagação. 2010. 79 f. Dissertação (Mestrado em Agronomia) - Universidade Federal da Amazônia, Belém, 2010. Disponível em: http://usadesign.com.br/mestrado/dissertacoes conlui das/herica oliveira.pdf. Acesso em 17 maio 2011.

PATANKAR, S. V. Computation of conduction and duct flow heat transfer. 1 ed. Maple Grove: Innovative Research, Inc., 1991. 340 p.

SANTOS, W. Métodos transientes de troca de calor na determinação das propriedades térmicas de materiais cerâmicos: I - o método de pulso de energia. Cerâmica, São Paulo, v. 51, p. 205-213, 2005. Disponível em: http://www.scielo.br/pdf/ce/v51n319/26793.pdf. Acesso em: 17 mar. 2011.

SCHMIDT, F. W.; HENDERSON, R. E.; WOLGEMUTH, C. H.. Introdução às ciências térmicas: termodinâmica, mecânica dos fluidos e transferência de calor. Tradução de José Roberto Simões Moreira. 1. ed. São Paulo: Edgard Blücher, 1996. 466 p. Título original: Introduction to thermal sciences: thermodynamics fluid dynamics heart transfer.

SOFFNER, M. de. L. A. P. Produção de polpa celulósica a partir de engaço de bananeira. 2001. $56 \mathrm{f}$. Dissertação (Mestrado em Ciências) - Escola Superior 
de Agricultura "Luiz de Queiroz", Universidade de São Paulo, Piracicaba, 2001. Disponível em: http://www.teses.usp.br/teses/disponiveis/11/11149/td e-13122001-114259/publico/soffner.pdf. Acesso em: 21 mar. 2011.

TARPANI, J. R.; ALMEIDA, E. G. R. de; SIMÊNCIO, E. C. A.; MOTA, L. P.; PAZ, J. H. A. A. Inspeção termográfica de danos por impacto em laminados de matriz polimérica reforçados por fibras de carbono. Polímeros: Ciência e Tecnologia, São Carlos, v. 19, n. 4, p. 318-328, 2009. Disponível em: http://www.scielo.br/pdf/po/v19n4/12.pdf. Acesso em: 17 mar. 2011.

TAVARES, S. G. Desenvolvimento de uma metodologia para aplicação de ensaios térmicos não destrutivos na avaliação da integridade de obras de artes. 2006. 169 f. Tese (Doutorado em Engenharia Mecânica) - Departamento de Engenharia Mecânica, Universidade Federal de Minas Gerais, Belo Horizonte, 2006. Disponível em: http://www.bibliotecadigital.ufmg.br/dspace/bitstream/1 843/SBPS-

7B4MPL/1/tese ufmg demec sinthya g. tavares.pdf. Acesso em: 19 mar. 2011.
TAVARES, S. G.; AGNANI, A.; ESPOSITO, E. FELIGIOTTI, M.; ROCCHI, S.; ANDRADE, R. M. de. Comparative study between infrared thermography and laser Doppler vibrometry apllied to frescoes diagnostic. In: QIRT - QUANTITATIVE INFRARED THERMOGRAPHY, 8., 2006, Padova. Anais... Padova: CNR-ITC, 2006. p. 1- Disponível em: http://qirt.gel.ulaval.ca/archives/qirt2006/papers/039.pd f. Acesso em: 17 mar. 2011. 